\title{
An Analysis of a Blockchain-Enabled E-Government Document Interchange Architecture (DIA) in Thailand
}

\author{
Chaiporn Thoppae, Prasong Praneetpolgrang \\ Faculty of Information Technology, Sripatum University, Thailand
}

\begin{abstract}
In 2008, the world was introduced to Blockchain. In 2009, the first open-source Blockchainbased cyber currency was introduced as Bitcoin. By 2025, studies indicate that Blockchain-based enterprise-wide application revenue will reach nearly $\$ 20$ billion. Therefore, this study proposes to build on the success of Thailand's National Single Window (THAINSW) gateway document interchange architecture (DIA) and further expand its availability to a much larger network of e-Government participants using Blockchain architecture. Therefore, the authors set how to investigate how and where Blockchain technology could be implemented in order to support of Thailand's 4.0 and digital economy visions. In addition to the qualitative analysis of the literature, the authors used input from a panel of 25 Thai experts to develop a foundation for the analysis based on Sathosi Nakamoto's seven Blockchain disciplines. This analysis was enhanced from the authors' own experiences in real-world Blockchain implementations. Results revealed that Blockchain is a highly innovative approach to secure transaction execution, information storage, performing functions, and establishing trust in an open architectural environment.
\end{abstract}

DOI: $10.18421 /$ TEM103-28

https://doi.org/10.18421/TEM103-28

Corresponding author: Chaiporn Thoppae,

Faculty of Information Technology, Sripatum University, Thailand.

Email: chaiporn.tho@spulive.net

Received: 18 March 2021.

Revised: 16 July 2021.

Accepted: 23 July 2021.

Published: 27 August 2021.

(c) BY-NC-ND (C) 2021 Chaiporn Thoppae \& Prasong Praneetpolgrang; published by UIKTEN. This work is licensed under the Creative Commons AttributionNonCommercial-NoDerivs 4.0 License.

The article is published with Open Access at www.temjournal.com
Blockchain has the potential to increase good governance, increase transparency and privacy, and reduce the chance of corruption.

Keywords - ASEAN Single Window (ASW), cryptocurrencies, distributed ledger technologies (DLT), proof-of-work (PoW).

\section{Introduction}

Distributed ledger technologies (DLT) such as Blockchain are processes that chronologically order cryptographically signed and expanding decentralized lists of irrevocable transactional records with total transparency to all network participants [1]. As such, network participants with access rights can trace back any historical transaction to any point in a transaction's history. Moreover, Blockchain value-exchange transactions execute directly from peer-to-peer (P2P), with algorithms consensually verifying each transaction.

However, in the conceptual development of using a Blockchain type concept for Bitcoin's digital currency in 2008 [2], one of the most widely known potential security vulnerabilities was the threat of double-spending [3]. One potential solution to a double-spending attack (DSA) was the creation of a bank-like central authority to verify and authorize transactions over the network.

However, in Bitcoin's decentralized Blockchain environment, Bitcoin's digital currency uses a consensus network and proof-of-work (PoW) concept to verify and execute payments with transaction digitally signatures. This service operates on top of the Bitcoin P2P network and verifies each transaction's order of execution is transparent to all Bitcoin network users. Moreover, by the use of a distributed time-stamping service, a DSA on a specific coin and the authorization of a single coin for two simultaneous transactions from two different users is prevented.

Furthermore, Blockchain technology is highly unique and innovative that transactions are broadcast to each node on the network whereupon the node creates a replicated version of events which 
precludes the need for an intermediary to facilitate each transaction. However, various researchers have pointed out that Blockchain's PoW can be considered inefficient due to the large amounts of energy that are required for the verification and storage processes. Thus the reason Bitcoin miners (transaction processing facilities) locate in cheap energy locations, such as geothermal abundant places such as Iceland [4].

Also, according to numerous studies, there are no clear consensus definitions as to what DLT and blockchain technologies are. In a similar manner as with the use of the word 'vehicle', which can cover an automobile, a container ship, or a space station, the term DLT also spans a broad set of technologies [5].

\subsection{Blockchains}

Blockchains are a series of multiple transactions which are clustered into a block which by highly sophisticated mathematical processes refer to the previous block. In 2018, it was estimated that Bitcoin's Blockchain contained 160 gigabytes worth of data from each previous Bitcoin wallet transaction [2].

Also in Bitcoin's case, a new block of previous transactions is created after a set time in which the newest block is validated across the network. From this process, a continuous chain of blocks is created. The Bitcoin-based blockchain was the first DLT mechanism used in cryptocurrency transactions. However, numerous alternatives have come into existence since then.

While Blockchain is technically a specific technology stack, over time the term has been increasingly applied to a loose confederation of DLT technologies and processes across a wide range of financial and business sectors [6], [7].

\subsection{The Consensus Mechanism (CM)}

A CM establishes trust in the accuracy of a data system transaction which was traditionally established by an administrator or intermediary in a centralized system such as a bank [1]. Also, the nodes in a DLT system use a CM to agree on the proposed transactions which ensure the data's consistency, integrity, and immutability [2].
Moreover, a P2P CM in a DLT uses governance rules and protocols to enable the recording, completion, and transaction execution. Therefore, a $\mathrm{CM}$ builds upon the previous transaction, forming a sequence of transactions, like a ledger.

\subsection{Smart Contracts (SCs)}

Another important element leveraged by multiple Blockchains is smart contracts, which is executable code that initiates a specified action based on the system's state or transaction [8]. Also, SCs operating within DLTs are independent and require no human administrator input, verification, or arbitration. Furthermore, the SC code ensures that double transaction conflicts are not included in the blockchain [7], which in some regards functions as a private regulatory framework of rules governing transactions between multiple parties [9].

Therefore, in addition to being able to handle improper operations or errors in code, SCs are also binding and irrevocable. Also, SCs reconcile transaction conflicts automatically with a new, valid transaction added only once to the blockchain, thus ensuring no double entries [10].

Therefore, this paper explores the slow yet incremental government approaches to the use of blockchain-type technologies which are still in their development phases [1]. However, Blockchain technology has shown great potential in transforming how government agencies and businesses process their transactions. Potentially, Blockchain visionaries hope that transaction efficiency, lower costs, and higher trust will be ensured.

However, the transformative power of Blockchain requires that the ecosystem and technological maturity of DLTs have to be increased to make accessible the technology's potential [6]. Therefore, government policy agendas should focus in the early stages of Blockchain's development on nontechnological barriers, such as existing legal and organizational frameworks, processes, and standards.

From an overview of the related literature concerning Blockchain-related technologies, the authors established two primary objectives for further research. These included:

1) An investigation of various approaches in the development of an architectural framework for electronic transaction document interoperability in Thailand;

2) The development of interoperability architecture for secure and efficient electronic document transactions using Blockchain to ensure the development of a sustainable digital economy in Thailand. 


\section{Literature Review}

\subsection{Blockchain Technology}

Blockchain is most probably the widest known form of technology in which a distributed ledger system is used [1]. Moreover, Blockchain's ledger system allows multiple forms of value-exchange transactions including cryptocurrencies, tokens, and information. Organized into blocks, Blockchain's digital signature uses a block data string, with both new and old blocks containing the same signature. Thus, P2P network blocks are immutably recorded using cryptographic trust and assurance mechanisms.

Furthermore, cryptocurrencies function as a decentralized subset of digital currencies. The P2P network uses protocols and algorithms to cryptographically create a highly secured payment mechanism acting as a medium of exchange, or a store of value [11], such as with Bitcoin. Therefore, a key benefit of Blockchain technology is that it offers a mechanism that is algorithm-based to manage and establish trust across numerous entities [1].

Therefore, due to Blockchain's self-executing SCs, nearly all transactional aspects in the domains of ecommerce, interbank communication, legal evidence timestamping, health, real estate, tourism, and transport need to be re-thought [12]. With algorithmic trust costs are likely to become lower as time goes by, Blockchain-based technologies have great potential when used for interactions between the public, businesses, and governments.

Fortunately, key generic Blockchain technical characteristics offer numerous benefits [13] within the domains already listed. These include:

1. One benefit is that a distributed ledger system shares content between multiple parties, which also ensures that each transaction is easily tractable and fully disclosable, even in large and complex ecosystems [14].

2. Another benefit is that the physical decentralization of transaction detail storage provides the elimination of a single point of failure, as compared to a single node vulnerable to cyber-attacks.

3. Also, in Blockchain transactions, new entries are appended to the previous transactions, which ensures the transaction and ledger integrity as it cannot be changed.

4. As verified transactions via a $\mathrm{P} 2 \mathrm{P} \mathrm{CM}$ ensure a transparent and accurate ledger [15], the need for a centralized authority to assure transaction validity is eliminated.

5. Finally, due to Blockchain's consensus mechanism and appended ledger transaction process, disintermediation is achieved eliminating the need for a broker-related intermediary and their related transaction costs [6], [12].

\subsection{Blockchain and Digital Objects}

In simple terms, Blockchains can be thought of as a decentralized database replicated across a global network of computers, which allows assets and their associated values to be represented by a digital value [16]. Moreover, with decentralization and the use of tokens, all forms of asset classes have the potential for Blockchain use. In this aspect, many have referred to Blockchain technology as potentially disruptive technology [17].

As previously stated, Bitcoin's blockchain was implemented as a decentralized currency system. However, Bitcoin is a software system executing a scripting language in a distributed environment, and Bitcoin is becoming the preeminent blockchainbased, decentralized, cryptocurrency system [7], [18]. Therefore, having the ability to track and verify digital transactions, Blockchain is becoming a contender for digital asset management systems and crypto-asset transactions [17].

\subsection{Blockchain and e-Government Policies}

Various case studies of e-Government concepts were analyzed by the authors to determine the complexity, costs, and success or failure of the respective e-Government implementations. One such study came from Russia in which the authors discussed the challenges of an administrative system's digital transformation through the lens of public administration [19]. In the Russian context, it was clearly stated that there was a discrepancy between plans and reality, with e-Government concepts and infrastructure realities creating a situation of stagnation, transparency, and control problems. However, the study did acknowledge that digital e-Government policies and the role of digitalization as an input or enabler of public policy modernization were potentially possible.

Another often cited reason for a Blockchain implementation was its unique ability to prevent officials' corruption as Blockchain's technology can provide governments and their citizens the ability to provide a tamper-proof record-keeping system that uses real-time transaction transparency, auditability, and automated SC functionality. However, it has to be noted that Blockchain-enabled systems have to be paired with a legal framework in which they can be effective. Technology alone cannot prevent corruption.

Furthermore, land title registries secured by Blockchain have been another frequently studied use for the technology. In Sweden, the main motivation was stated to be an increase in efficiency [20]. In other locals such as Honduras and India, Blockchain implementations were studied as a way to instill and 
expand property rights, while also adding transparency to a highly corrupt system.

Finally, in Thailand's real estate sector, Blockchain is being considered as a way to 'tokenize' large project property investments across a broad spectrum of potential investors. Tokenizing property projects have the potential to link ownership and its value, to a digital token.

\subsection{Thai Document Interchange Architecture (TDIA)}

Furthermore, to facilitate e-Government transparency, the authors propose the efficient and highly secure Blockchain-based integration between the existing Thailand National Single Window (THAINSW) information system gateway (Thailand Gateway) and its existing Thai FDA (Food \& Drug Administration) and Thai Customs' users. Also, the THAINSW can serve as a doorway for investment, trade, citizens, and tourists who need to transfer secured documents (such as Covid-19 vaccine shot records) through the secure electronic transaction (etransactions) document interchange architecture (DIA) based on Blockchain technology [23].

Moreover, the proposed Blockchain-enabled THAINSW and DIA would increase processing and transmission time, while reducing Thai governmental operating costs. A Blockchain e-Government enabled system could also increase job tracking while facilitating document transfer between both domestic and international agencies. This would then encourage the government to business (G2B) data integration such as in custom's clearance information and business to business (B2B) data integration [22].

Also, a blockchain-enabled THAINSW the potential to simplify further international trade transaction processes and documents. Additional benefits are the ability to significantly reduce the number of processes, documentation, and time required for completing Custom's regulatory border requirements. Thus far, studies have indicated that the THAINSW is already saving Thailand's logistic enterprises over $\$ 1.5$ billion annually [23].

Therefore, e-Government policies should be focused on providing innovative, agile, and corruption-free public services (government agencies to citizens or G2C). E-Government architectures should facilitate direct communication between public agencies, a nation's citizenry, and economic entities. On the most basic level, public information registration and exchange processes should be improved. The decentralization of Blockchains is at the core of reshaping the way governments interact with their citizens and with each other. Finally, these services and their delivery models potentially could leverage e-Government digital technologies and assets with citizen information. This represents a paradigm shift in e-Government policy making and service delivery (Figure 1).

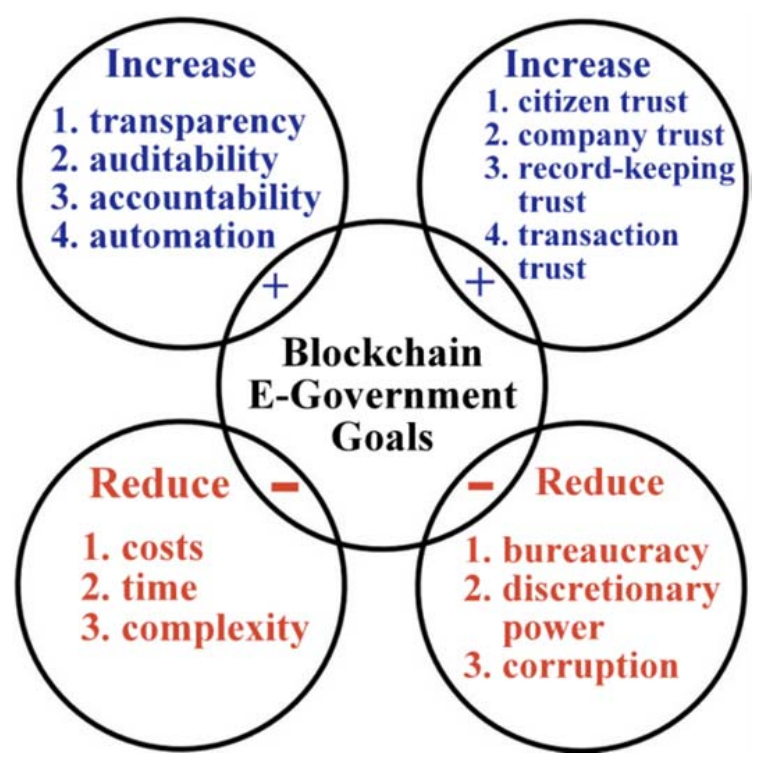

Figure 1. Blockchain E-Government goals Sources: [6], [21]

\section{Methods}

\subsection{Qualitative Analysis}

Qualitative analysis was conducted by use of a focus group to obtain in-depth interviews from 25 individuals concerning development guidelines for a Thai secure electronic transaction DIA framework based on blockchain technology. Each member of the focus group had expertise in e-transactions, network architectures, cybersecurity, digital economy, or blockchain technologies. The experts were also executives or specialists in their related fields and had a minimum of five years of related work experience. Furthermore, there were five chief information officers (CIOs) from five different Thai ministries.

\subsection{Collection Method}

Purposive sampling was used to select each of the 25 individuals for the study. Respondents were asked to respond to the qualitative documentary data concerning education, law, regulation, national strategy, national policy, literary, and research policies related both domestically and internationally to a secure, Blockchain-based DIA.

\subsection{Data Analysis}

The qualitative analysis of the data was conducted in three main stages. This included data synthesis and reduction, data organization, and data interpretation. 


\section{Results}

\subsection{European Interoperability Framework (EIF)}

From the Thai experts' input from the focus groups concerning e-government and e-transactions in a highly secured Blockchain-enabled architectural framework, it was suggested that the authors should look to the European Union (EU) European Interoperability Framework (EIF) as a guideline for a DIA architecture framework. From the EIF documents, it is reported that the single greatest obstacle to the integration of a single digitally based market is the lack of interoperability [23], which is rooted in the local areas' bottom-up problem solution mechanism which has arisen from their continued use of legacy systems. This has resulted in the fragmentation of ICT resources into non-compatible islands which are highly complex, interoperable environments.

However, it is suggested that there are six steps to achieving digital interoperability. These include the identification, assessment, implementation, monitoring, change management, and documentation of potential candidate specifications and standards. Problematically, however, is the lack of in-house technical skills and personnel. Finally, a holistic approach to interoperability governance is also critical as it integrates all the resources needed to apply it.

\subsection{United Nations Centre for Trade Facilitation and Electronic Business (UN/CEFACT)}

Additional input from the panel of Thai experts suggested that guidelines from the UN/CEFACT were useful to examine in a Thai Blockchain DIA implementation through the existing THAINSW. From these sources, the UN/CEFACT's Modeling Methodology 2.0 (UMM 2.0) provided an excellent guideline for inter-organizational system development and document exchange architectures [24]. It should be noted here that the UN/CEFACT Recommendation 33 was used in the conceptualization of the ASEAN Agreement to Establish an ASEAN Single Window gateway. This was the linage to the launching in 2008 of the THAINSW and the proposed Blockchain secured DIA architecture/gateway in this study.

\subsection{Blockchain-Enabled Thai E-Government Network and THAINSW Gateway}

From the inception and implementation of the THAINSW into the ASEAN ASW system, document security, authentication, and tracking has been a primary consideration. In recent years, the scope of these early precepts of a 'digital economy' has greatly expanded in Thailand, with the Kingdom has become an innovator in the field under a newly created Ministry of Digital Economy and Society (MDES). Moreover, a new National Digital Economy Master Plan (NDEMP) has been created which details the Kingdom's 'five key pillars' for a new digital economy [25]. Included in Pillar 3 is the stated need for public confidence assurance through a digital technology architecture that ensures cybersecurity through verifiable transactions and identities, which also helps in Thailand's fight against corruption. Therefore, Figure 2 is presented as an overview by which users and agencies could potentially participate in a Thai e-Government Blockchain DIA implementation.

In Figure 3, the authors outline the file transfer service infrastructure and process within a Blockchain-enabled DIA. This is consistent with other studies in Thailand in which similar enterprisewide The Open Group Architecture Framework $\left(\right.$ TOGAF $\left.^{\circledR}\right)$ Version 9.2 and Analytic Hierarchy Process (AHP) information system architectures have been suggested [26]. Specifically, TOGAF $^{\circledR} 9.2$ has its roots in the US Department of Defense which today is a freely available integrated hierarchy of architectures for larger organizational teams operating in overarching architectural governance models.

Furthermore, in Figure 3 the authors have diagramed the e-transaction switching architecture and message/file delivery process through the proposed Blockchain DIA encrypted networks.

In moving from left to right, in Figure 3 we see where an 'Other Government Agency' (OGA) staff member using an encrypted SSL/TSL (Secure Socket Layer/Transport Layer Security) Internet protocol moves an $X M L$ (Extensible Markup Language) encoded document into the organization's database (file) management system (DMS). At this point, the generic message is merged with the message sender's private encryption key and once again using an SSL/TSL connection, transfers the file into the external 'cloud' (Internet/Extranet). Moving through the cloud routers using SOAP (Simple Object Access Protocol) to the destination OBA (Other Business Agency), the process is reversed and the message is decrypted for the final end-user. 


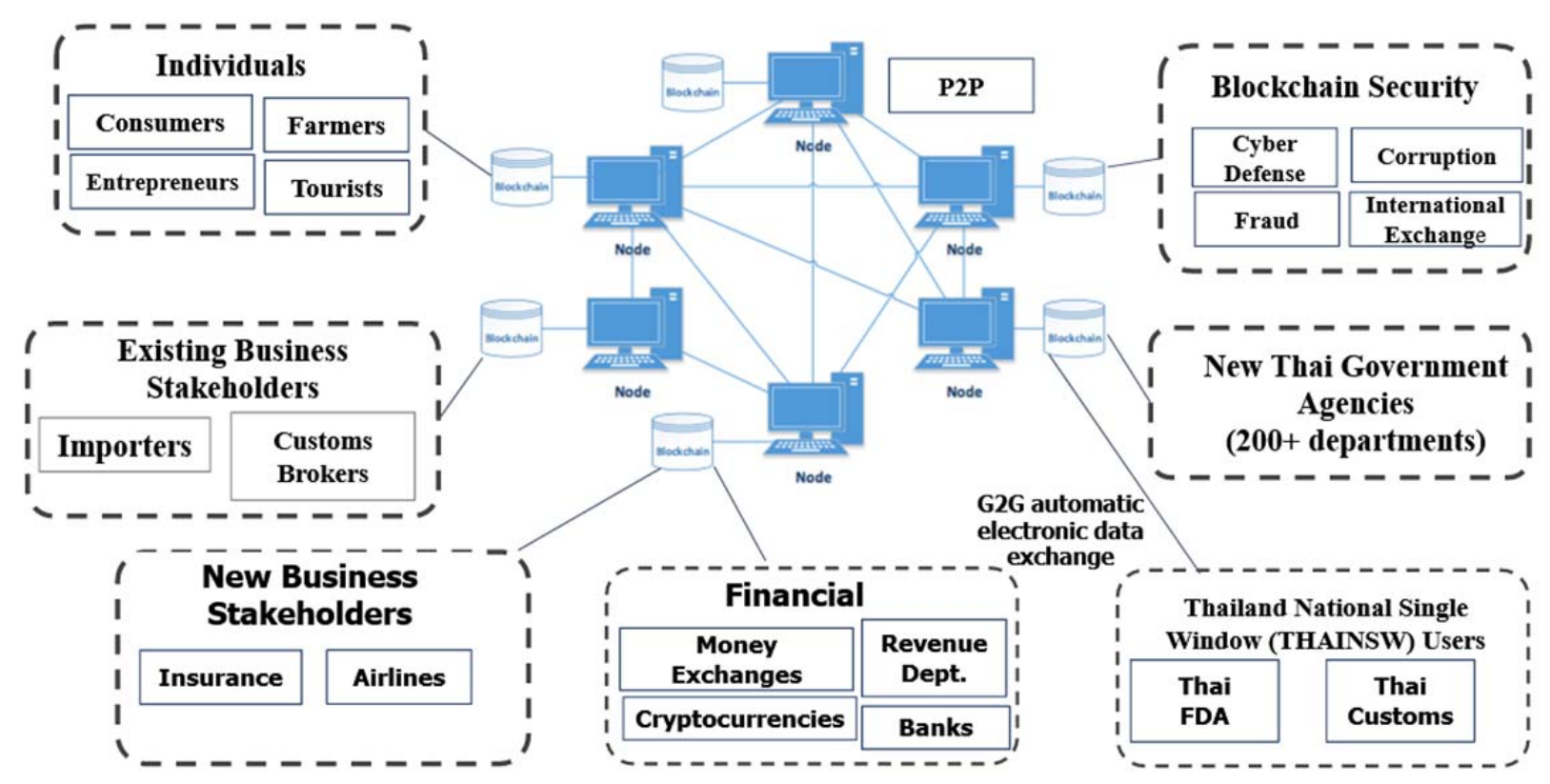

Figure 2. Proposed Blockchain-enabled Thai e-Government network and THAINSW Gateway Source: The authors

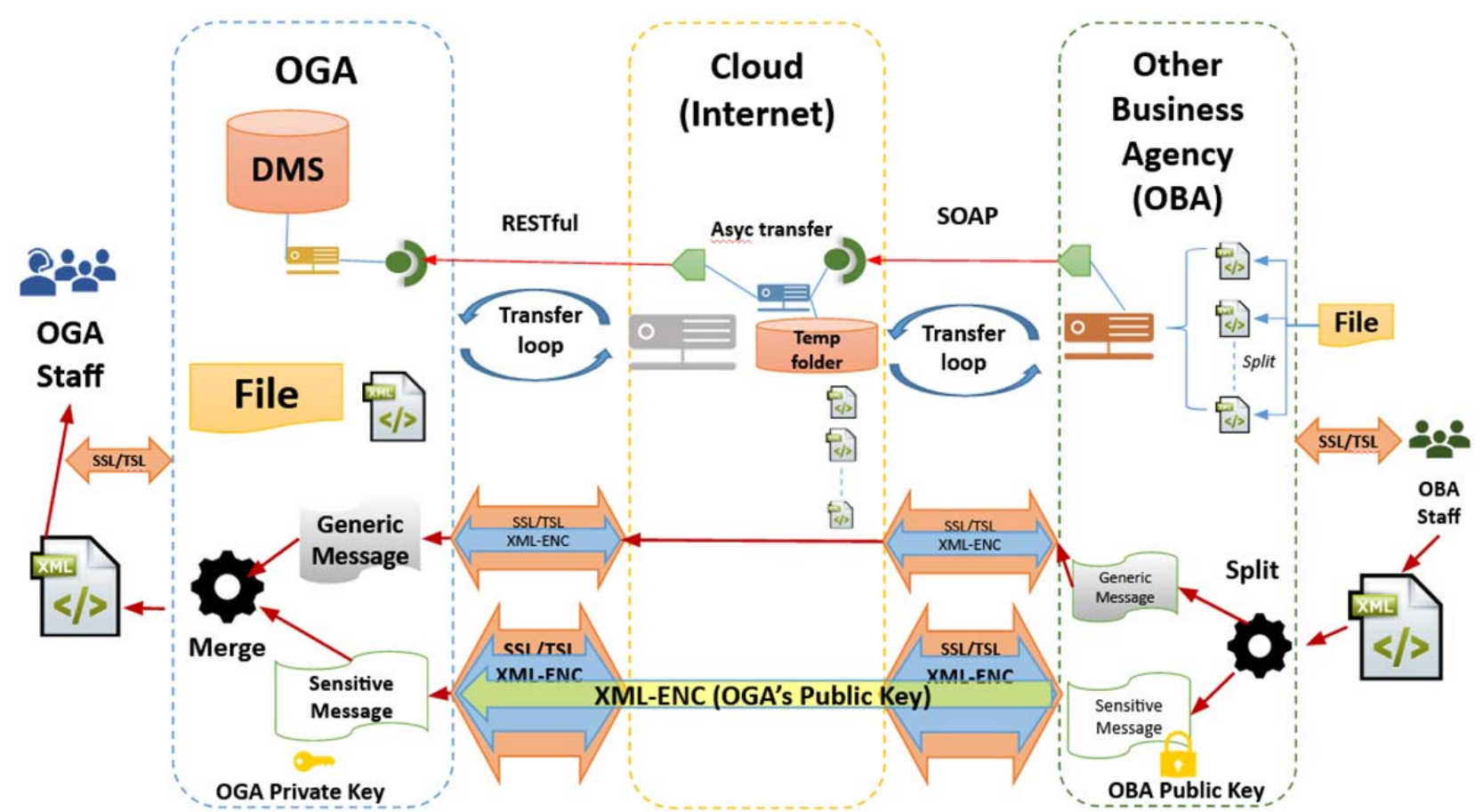

Figure 3. Thai e-Government DIA Blockchain-enabled network message and file flow diagram Source: The authors

\section{Discussion}

Rising from concepts as early as 1991, and after being combined with the Markle Tree concept [27], [28], someone using the name 'Sathosi Nakamoto' created a cryptocurrency called 'Bitcoin' in 2008 using Blockchain as its foundation [2]. From Nakamoto's documents, seven design principles were extracted as being the foundations in blockchain implementation. These were 1) networked integrity, 2) distributed power, 3) value as an incentive, 4) security, 5) privacy, 6) rights preserved, and finally, 7) inclusion [2], [29].

Moreover, this study determined that government policy support is essential as well in Thailand. This is consistent with the establishment of Thailand's Ministry of Digital Economy and Society and the new National Digital Economy Master Plan working within the earlier government goals for a Thailand 4.0 based economy [23], [25]. 
When the study focused on strategic policy, it was concluded that a joint, sector-wide development policy was necessary. Also, public data should be easily accessible and transparent, with the proposed Blockchain DIA helping to reduce time and productivity losses while creating opportunities for the users and agencies involved. An excellent example of effective use of a national DIA is the billions of dollars logistics companies have saved since the establishment of the THAINSW into the ASEAN ASW system [23].

When examining the reasons and mechanisms for an efficient and secure DIA, it was determined that cost justification was a primary reason as well. This was due to the continuing loss in the Kingdom's productivity due to official corruption, lack of transaction transparency, wasted time, and poor governance. However, numerous foreign studies and domestic Thai experts believe that Blockchain type technology has the potential to minimize these problems.

Also, from the authors' participation in multiple real-world Blockchain test sites in Thailand, it was established that network integrity validation and verification comes from the use of an XML schema in which the XML documents could be electronically signed, tracked, and verified. Authority for electronically signed documents has also been written into Thai law which on April 15, 2019, was authorized with Thailand's Electronic Transaction Act B.E. 2544 (2001) (ETA).

Further integrity in a Blockchain DIA is assured by the use of international standards, message identification mechanism, encryption, message verification and completeness, and the ability to detect and prevent message rejection. Certificates for all e-transactions are also done automatically, with message and file logs also making use of encryption. Also, making use of a distributed storage system [30] with independent backup systems helps ensure data survivability from network hacker attacks or critical infrastructure failure from floods, fires, etc.

Also, by elimination of 'intermediaries' in the workflow process, corruption is reduced, greater fairness is achieved, and society as a whole benefits [2], [29]. However, further data integrity and safety procedures should still require the use of digital signatures and TSL level communications between systems (Figure 2). Therefore, no single process or device 'secures' the network; it is a combination of these that secures the data and messages within the Blockchain-enabled DIA.
Furthermore, from the authors' participation in the Thai Food and Drug Administration (FDA) Blockchain test site, results revealed that a decentralized system in which responsibility and authority were trusted down to the departmental level worked well. Moreover, there were clear management benefits to a Blockchain DIA, which was supported by the use of a fee-based system from outside contractors.

\section{Conclusion}

Research shows that Blockchain is a technology highly suitable for developing a secure document interchange architecture framework for etransactions. Moreover, each transaction is embedded in blocks that are mathematically linked to a previous block and encrypted. This combination of techniques makes transactions and blocks immutable. However, although there are growing pains in a Blockchain implementation (e.g., healthcare, land registry, customs), the architecture is suitable across a wide spectrum of transactions and industries. It is also very suited for the implementation in existing Gateways such as the THAINSW being used by logistics and customs agencies. Blockchain has the potential to increase good governance, increase transparency and privacy, and reduce the chance of corruption.

\section{Acknowledgement}

This research was supported by funding from the Office of the National Research Council of Thailand (NRCT) for graduate studies for the fiscal year 2019.

\section{References}

[1]. Alam, B., Thakur, V., \& Singh, S. (2021). Key management for blockchain technology. ICT Express, 7(1), 76-80.

[2]. Nakamoto, S. (2008). Bitcoin: A peer-to-peer electronic cash system. Decentralized Business Review, 21260.

[3]. Rubasinghe, I. D., \& De Zoysa, T. N. K. (2012). Transaction verification model over double spending for peer-to-peer digital currency transactions based on blockchain architecture. International Journal of Computer Applications, 975, 8887.

[4]. Hearn, A. (2018, February 14). How Iceland became the Bitcoin miner's paradise. The Guardian.

[5]. R. H. Campbell Rab, Thompson Gillian, Ferry Peter, "Distributed Ledger Technologies in Public Services," no. June, 2018.

[6]. Allessie, D., Sobolewski, M., Vaccari, L., \& Pignatelli, F. (2019). Blockchain for digital government. Luxembourg: Publications Office of the European Union. 
[7]. Tasca, P., \& Tessone, C. (2019). A Taxonomy of Blockchain Technologies: Principles of Identification and Classification. Ledger (Pittsburgh), 4, 1-39.

[8]. Alharby, M., Aldweesh, A., \& van Moorsel, A. (2018, November). Blockchain-based smart contracts: A systematic mapping study of academic research (2018). In 2018 International Conference on Cloud Computing, Big Data and Blockchain (ICCBB) (pp. 1-6). IEEE.

[9]. Ellul, J., Galea, J., Ganado, M., Mccarthy, S., \& Pace, G. J. (2020, October). Regulating Blockchain, DLT and Smart Contracts: a technology regulator's perspective. In ERA Forum (Vol. 21, No. 2, pp. 209220). Springer Berlin Heidelberg.

[10]. Clack, C. D., Bakshi, V. A., \& Braine, L. (2016). Smart contract templates: foundations, design landscape and research directions. arXiv preprint arXiv:1608.00771.

[11]. Alqassem, I., \& Svetinovic, D. (2014, September). Towards reference architecture for cryptocurrencies: Bitcoin architectural analysis. In 2014 IEEE International Conference on Internet of Things (iThings), and IEEE Green Computing and Communications (GreenCom) and IEEE Cyber, Physical and Social Computing (CPSCom) (pp. 436443). IEEE.

[12]. Quiniou, M. (2019). Blockchain: the advent of disintermediation. John Wiley \& Sons.

[13]. Zheng, Z., Xie, S., Dai, H., Chen, X., \& Wang, H. (2017, June). An overview of blockchain technology: Architecture, consensus, and future trends. In 2017 IEEE international congress on big data (BigData congress) (pp. 557-564). IEEE.

[14]. Hull, R., Batra, V. S., Chen, Y. M., Deutsch, A., Heath III, F. F. T., \& Vianu, V. (2016, October). Towards a shared ledger business collaboration language based on data-aware processes. In International conference on service-oriented computing (pp. 18-36). Springer, Cham.

[15]. Raikwar, M., Gligoroski, D., \& Kralevska, K. (2019). SoK of used cryptography in blockchain. IEEE Access, 7, 148550-148575.

[16]. De Kruijff, J., \& Weigand, H. (2017, June). Understanding the blockchain using enterprise ontology. In International Conference on Advanced Information Systems Engineering (pp. 29-43). Springer, Cham.

[17]. Laurent, P., Chollet, T., Burke, M., \& Seers, T. (2018). The tokenization of assets is disrupting the financial industry. Are you ready. Inside magazine, 19, 62-67.
[18]. Pillai, B, Biswas, K, \& Muthukkumarasamy, V. (2019). Blockchain interoperable digital objects. In J. Joshi, S. Nepal, Q. Zhang, \& L. J. Zhang (Eds.), Blockchain - ICBC 2019. ICBC 2019. Lecture Notes in Computer Science, 11521. Springer.

[19]. Gritsenko, D., \& Zherebtsov, M. (2021). EGovernment in Russia: Plans, Reality, and Future Outlook. In The Palgrave Handbook of Digital Russia Studies (pp. 33-51). Palgrave Macmillan, Cham.

[20]. Bennett, R., Miller, T., Pickering, M., \& Kara, A. K. (2021). Hybrid approaches for smart contracts in land administration: Lessons from three blockchain proofsof-concept. Land, 10(2), 220.

[21]. Alipour-Hafezi, M., Horri, A., Shiri, A., \& Ghaebi, A. (2010). Interoperability models in digital libraries: an overview. The Electronic Library, 28(3), 438-452.

[22]. Aras, S. T., \& Kulkarni, V. (2017). Blockchain and its applications-a detailed survey. International Journal of Computer Applications, 180(3), 29-35.

[23]. Pruksasri, P., vd Berg, J., \& Keretho, S. (2011). Accountability in Single Window systems using an Internal Certificate Authority: A case study on Thailand's National Single Window system. In Proceeding of the 5th IADIS Multi conference on computer science and information systems (pp. 129136).

[24]. Huemer, C., Liegl, P., Motal, T., Schuster, R., \& Zapletal, M. (2008, August). The development process of the UN/CEFACT modeling methodology. In Proceedings of the 10th international conference on Electronic commerce (pp. 1-10).

[25]. Collen, M. F., \& Kulikowski, C. A. (2015). The Development of Digital Computers. In The History of Medical Informatics in the United States (pp. 3-73). Springer, London.

[26]. Wanotayapitak, S., \& Wannapiroon, P. (2018). Design and Assessment of System Architecture in Automatic System Transformation of E-Portfolio to E-Assessment through TOGAF and AHP. Progress in Applied Science and Technology, 8(2), 96-124.

[27]. Merkle, R. C. (1987, August). A digital signature based on a conventional encryption function. In Conference on the theory and application of cryptographic techniques (pp. 369-378). Springer, Berlin, Heidelberg.

[28]. Haber, S., \& Stornetta, W. S. (1991). How to timestamp a digital document. Journal of Cryptology, 3(2), 99-111.

[29]. Tapscott, D., \& Tapscott, A. (2018). Blockchain revolution: How the technology behind Bitcoin and other cryptocurrencies is changing the world. Penguin Pub.

[30]. Swan, M. (2015). Blockchain: Blueprint for a new economy. " O'Reilly Media, Inc.". 